\title{
Ferrets further disease research
}

\section{SCIENTIFIC NAME}

Mustela putorius furo

TAXONOMY

PHYLUM: Chordata

CLASS: Mammalia

ORDER: Carnivora

FAMILY: Mustelidae

\section{Physical description}

Domestic or European ferrets are carnivorous mammals related to weasels, minks, otters and skunks. They have long bodies, short legs and thick fur with various color patterns. The natural pattern, called sable or fitch, features a yellowish coat with dark patches over the limbs and tail, a facial mask and dark brown eyes. Ferrets have anal scent glands and sebaceous glands but lack sweat glands and hence are particularly susceptible to heat ${ }^{1}$. Adult males weigh $\sim 2 \mathrm{~kg}$, whereas females are smaller at $\sim 1 \mathrm{~kg}$. Ferrets live for roughly $6-8$ years.

\section{Research résumé}

Ferrets have been used as research models since the early 1900s (ref. 2).

They are hardy animals that thrive in the lab, provided that their social behaviors, diet and other habits, such as their inclination to burrow and hide, are accommodated.

Despite a relatively small representation in the laboratory, ferrets have a distinct niche in biomedical research. Their tracheal and lung anatomies, which, like their narrow, elongated bodies and short legs, are adapted for burrowing, afford decreased airway resistance and a relatively large lung capacity that facilitate respiratory research. Because lung morphology is similar between ferrets and humans, ferrets are used in the study of cystic fibrosis ${ }^{3}$.

In addition to having similar respiratory anatomy, ferrets are also susceptible to respiratory diseases that affect humans (notably, influenza and severe acute respiratory syndrome (SARS)-associated corona virus) and often show clinical signs similar to those observed in humans. Influenza research has become especially important in recent years owing to the emergence of highly pathogenic strains of influenza that are global health threats. Ferrets are the model of choice for developing influenza vaccines and therapeutics ${ }^{4}$.

Ferrets are also used to model human gastrointestinal disease, again because of their anatomical and physiological similarities to humans, as well as the fact that both species host Helicobacter organisms that cause gastritis. A ferret model for peptic ulcer disease has aided studies of the epidemiology of this disease as well as its pathogenesis and treatment ${ }^{2}$. Ferrets have also been used to study the emetic potential of drugs ${ }^{5}$ and to develop anti-emetic medications ${ }^{6}$, such as those given to cancer patients.

\section{Regulatory coverage}

Despite their importance in biomedical research, ferrets are not specifically mentioned in the US Animal Welfare Act and Regulations ${ }^{7}$ or in many of the commonly used recommendations and guidelines for laboratory animal care. They are covered as 'other animals' under Subpart F of the Animal Welfare Act, which lists only general information on facilities and husbandry and does not include specific requirements for cage size, feed composition or temperature, among other parameters. The ferret is also covered under the Public Health Service Policy on Humane Care and Use of Laboratory Animals ${ }^{8}$. Ferrets are not specifically addressed in the most recent edition of the Guide for the Care and Use of Laboratory Animals ${ }^{9}$, but the European Convention for the Protection of Vertebrate Animals Used for Experimental and Other Scientific Purposes does include a section dedicated to husbandry and housing requirements for ferrets ${ }^{10}$.
1. Matchett, C.A., Marr, R., Berard, F.M., Cawthon, A.G. \& Swing, S.P. The Laboratory Ferret (CRC, Boca Raton, FL, 2012).

2. Ball, R.S. Issues to consider for preparing ferrets as research subjects in the laboratory. ILAR J. 47, 348-357 (2006).

3. Fisher, J.T. et al. Comparative processing and function of human and ferret cystic fibrosis transmembrane conductance regulator. J. Biol. Chem. 287, 21673-21685 (2012).

4. Banner, D. \& Kelvin, A.A. The current state of H5N1 vaccines and the use of the ferret model for influenza therapeutic and prophylactic development. J. Infect. Dev. Ctries. 6, 465-469 (2012).

5. Soref, C.M., Hacker, T.A. \& Fahl, W.E. A new orally active, aminothiol radioprotector-free of nausea and hypotension side effects at its highest radioprotective doses. Int. J. Radiat. Oncol. Biol. Phys. 82, e701-e707 (2012).

6. Duffy, R.A. et al. Rolapitant (SCH 619734): a potent, selective and orally active neurokinin NK1 receptor antagonist with centrally-mediated antiemetic effects in ferrets. Pharmacol. Biochem. Behav. 102, 95-100 (2012).

7. Animal Welfare Act Regulations. Code of Federal Regulations. Title 9, Chapter 1.

8. Public Health Service. Policy on Humane Care and Use of Laboratory Animals (US Department of Health and Human Services, Washington, DC, 1986, amended 2002).

9. Institute for Laboratory Animal Research. Guide for the Care and Use of Laboratory Animals 8th edn. (National Academies Press, Washington, DC, 2011).

10. Council of Europe. European Convention for the Protection of Vertebrate Animals Used for Experimental and Other Scientific Purposes ETS 123. 1986. 